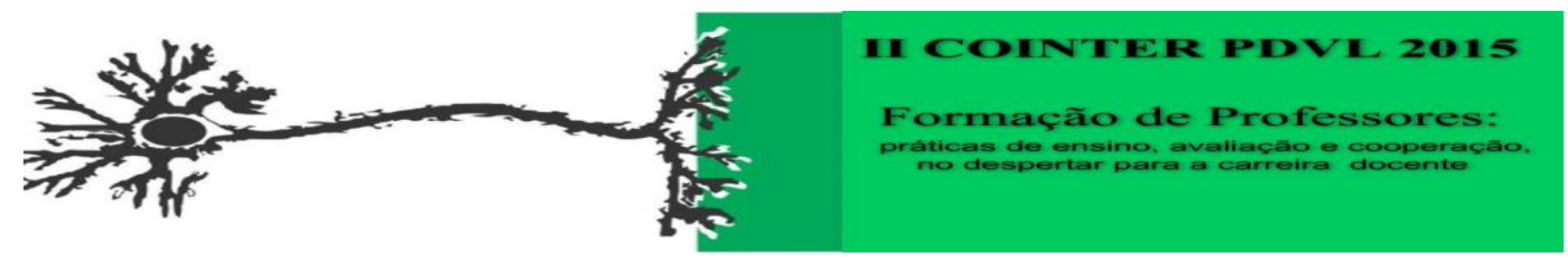

\title{
ANÁLISE COMPARATIVA DAS MATRIZES CURRICULARES DO CURSO DE LICENCIATURA EM GEOGRAFIA NO IFPE E NA UNLP
}

\author{
Apresentação: Pôster
}

\begin{abstract}
Matheus Souto Mendes de Azevêdo ${ }^{1}$; Alessandro Augusto Guerra Junior ${ }^{2}$; Adauto Gomes Barbosa $^{3}$
\end{abstract}

\section{Introdução}

O Programa Internacional Despertando Vocações para a Licenciatura (PDVL) possui o caráter extensionista, além de possuir questões intimamente relacionadas a ensino e pesquisa. $\mathrm{O}$ trabalho exposto aqui retrata um estudo realizado na Falcutad de Humanidades y Ciências de la Educación (Fahce), da Universidad Nacional de La Plata (UNLP), localizada em La Plata - Buenos Aires (Argentina). Estudamos o segundo semestre desse ano (2015.2) nessa universidade, e realizamos um estudo comparativo. Tendo em vista que o modelo educacional argentino, tanto no Ensino Básico, quanto no Ensino Superior se distingue bastante do modelo educacional brasileiro, podemos concluir que as matrizes curriculares do curso de Licenciatura em Geografia (Na Argentina, denominado "Professorado em Geografia") apresentam variadas distinções. Porém, apesar das diferenças, podemos notar também algumas semelhanças entre os dois sistemas educacionais. Esse estudo se faz de suma importância para entendermos os mecanismos de políticas educacionais nesse sentido de relação Brasil-Argentina.

\section{Fundamentação Teórica}

Sendo assim, o referencial teórico-metodológico requer uma atividade extensionista para além de algo essencialmente prático. Sempre foi pensado em se realizar ações de modo que se priorize a relação entre teoria e prática, para assim, desenvolvermos uma metodologia de trabalho que possa, ao abordar o campo de extensão, considerá-lo a partir das relações históricas e sociais,

\footnotetext{
${ }^{1}$ Licenciatura em Geografia/Instituto Federal de Pernambuco (IFPE)/ Programa de Extensão Internacional Despertando Vocações para as Licenciaturas - PDVL/ azevêdomatheus83@gmail.com

${ }^{2}$ Licenciatura em Geografia/Instituto Federal de Pernambuco (IFPE)/ Programa de Extensão Internacional Despertando Vocações para as Licenciaturas - PDVL/ augguerra@gmail.com

${ }^{3}$ Doutor em Geografia/Instituto Federal de Pernambuco (IFPE)/ Programa de Extensão Internacional Despertando Vocações para as Licenciaturas - PDVL/adauto@recife.ifpe.edu.br
} 
que favorecem os processos de produção e reprodução da existência do ser social. Desse modo, é importante um referencial teórico-metodológico que perceba o processo de extensão como totalidade, que requeira tomar os seus diversos aspectos em estreita relação de dependência e de determinações recíprocas. Foi elaborada uma abordagem apoiada em Paulo Freire, trabalhando com uma perspectiva de educação crítico-reflexiva e transformadora, de compreensão e apropriação do movimento da realidade. Como o próprio Freire, afirma, deve-se haver uma perspectiva de transformação social, ao compreender a educação como um processo sócio-político. Além de aspectos naturais, deve-se trabalhar também com a interdisciplinaridade, onde Lana de Souza Cavalcanti realiza estudos simples e eficazes, e ter uma visão ampla e crítica do estudo extensionista proposto, sempre lembrando de uma característica social.

\section{Metodologia}

Para o desenvolvimento do trabalho, realizou-se um estudo de caso, que diz respeito a uma pesquisa que visa comparar, buscando as particularidades da matriz curricular do curso de Licenciatura em Geografia em duas diferentes instituições: IFPE (Brasil) e FAHCE (Argentina). A pesquisa é de caráter comparativo, pois relata as realidades de cada curso. Nos currículos, foram identificadas as disciplinas, e a partir dessas disciplinas, foram realizadas reflexões acerca desse tema.

\section{Resultados e Discussões}

Para começar, notamos que o tempo de duração de curso é diferente. Enquanto no IFPE, o curso de Licenciatura em Geografia dura quatro anos, o curso de Professorado em Geografia na FAHCE dura cinco anos. Uma particularidade muito interessante da FAHCE é que as matérias se subdividem em duas: Teóricas e Práticas. Por exemplo, a matéria de "Metodologia Científica" se dividiria em "Metodologia Científica Teórica" e Metodologia Científica Prática", cada uma com um(a) professor(a) diferente. Uma matéria pode ter até quatro professores. Na maioria, as matérias do curso na FAHCE são optativas. Em síntese, o obrigatório é que se curse no mínimo cinco anos, e que as matérias obrigatórias sejam cursadas. A distribuição das matérias por ano na matriz curricular da FAHCE é um sistema sugestivo, pois o aluno tem a liberdade de montar sua grade curricular. 


\begin{tabular}{|c|c|c|c|c|c|c|c|}
\hline Per. & Sigla & Componente Curricular & C.H. & créd. & Pré-requisitos & Co-requisitos: & Habilitacso \\
\hline \multirow{6}{*}{1} & CGRC.006 & Fundamentos da Ciencia Geográfica & 67.5 & 5 & & & \\
\hline & CGRC.005 & Fundamentos de Geologia & 67.5 & 5 & & & \\
\hline & CGRC.DO4 & Fundamentos Sociológicos da Educaçáo & 54 & 4 & & & \\
\hline & CGRC.002 & Introduçäo a Filosofia & 40.5 & 3 & & & \\
\hline & CGRC.001 & Metodologia Cientifica & 54 & 4 & & & \\
\hline & CGRC.003 & Organizaçăo e Gestáo da Educaçäo Brasileira & 54 & 4 & & & \\
\hline \multirow{6}{*}{2} & CGRC.016 & Oimatologia & 67.5 & 5 & & & \\
\hline & CGRC. 007 & Didática & 54 & 4 & & & \\
\hline & CGRC.OOB & Fundamentos Históricos e filosóficos da Educaçáo & 54 & 4 & & & \\
\hline & CGRC.010 & Geografia Econỏmica & 54 & 4 & & & \\
\hline & CGRC.DO9 & Geomorfologia & 54 & 4 & CGRC.005 & & \\
\hline & CGRC.012 & Histúria do Pensamento Geografico & 54 & 4 & CGRC. 006 & & \\
\hline \multirow{6}{*}{3} & CGRC.013 & Antropologia Cultural & 54 & 4 & & & \\
\hline & CGRC.011 & Cartografia Básica & 54 & 4 & & & \\
\hline & CGRC.014 & Formacáo Económica e Territorial do Brasil & 54 & 4 & & & \\
\hline & CGRC. 015 & Fundamentos Psicolígicos da Educaçäo & 67.5 & 5 & & & \\
\hline & CGRC.017 & Geografia da Populaçá & 54 & 4 & & & \\
\hline & CGRC. 018 & Metodologia do Ensino de Geografia & 54 & 4 & CGRC. 007 & & \\
\hline \multirow{6}{*}{4} & CGRC.020 & Biogeografia & 54 & 4 & & & \\
\hline & CGRC. 021 & Geografia e Cultura & 54 & 4 & & & \\
\hline & CGRC.022 & Geografia Politica & 54 & 4 & & & \\
\hline & CGRC.019: & Geoprocessamento & 54 & 4 & CGRC. 011 & & \\
\hline & CGRC.024 & Laboratório e Prática do Ensino de Geografia I & 67.5 & 4 & & & \\
\hline & CGRC.023 & Pedologia e Edafologia & 54 & 4 & & & \\
\hline \multirow{6}{*}{5} & CGRC.030 & Estágio Supervisionado I & 94.5 & 7 & & & \\
\hline & CGRC. 025 & Estatistica Aplicada a Geografia & 40.5 & 3 & & & \\
\hline & CGRC. 026 & Geografia Agrária & 54 & 4 & & & \\
\hline & CGRC.028 & Geografia das Indústrias e Servigos & 40.5 & 3 & & & \\
\hline & CGRC. 027 & Geografia Regional do Mundo & 40.5 & 3 & & & \\
\hline & CGRC.029 & Laboratório e Prática do Ensino de Geograria II & 67.5 & 5 & CGRC. 024 & & \\
\hline \multirow{6}{*}{6} & CGRC.031 & Avaliaçä Educacional & 54 & 4 & & & \\
\hline & CGRC.035 & Deservolvimento e Meis Ambiente & 40.5 & 3 & & & \\
\hline & CGRC. 036 & Estágio Supervisionado II & 94.5 & 7 & CGRC. 030 & & \\
\hline & CGRC.033 & Geografia Regional do Brasil & 40.5 & 3 & & & \\
\hline & CGRC. 032 & Geografia Urtana & 54 & 4 & & & \\
\hline & CGRC.034 & Métodos e Técnicas da Pesquisa en Geografia & 54 & 4 & & & \\
\hline \multirow{6}{*}{7} & CGRC.042 & Estágio Supervisionado III & 108 & 8 & CGRC. 0.36 & & \\
\hline & CGRC. 040 & Geografia do Nordeste Brasileiro & 40.5 & 3 & CGRC. 033 & & \\
\hline & CGRC. 039 & Hidrogeografia & 40.5 & 3 & & & \\
\hline & CGRC. 037 & Mundia lizaçäo e Saciedade de Consumo & 40.5 & 3 & & & \\
\hline & CGRC. 041 & Pesquisd em Ensifo de Geografia & 54 & 4 & CGRC.034 & & \\
\hline & CGRC. 038 & Seminário Interdisciplinar & 54 & 4 & & & \\
\hline \multirow{5}{*}{8} & CGRC. D46 & Estágio Supervisionado IV & 108 & 8 & CGRC.042 & & \\
\hline & CGRC.043 & Estratígias de Educaçáo Ambiental & 40.5 & 3 & & & \\
\hline & CGRC.045 & Libras & 54 & 4 & & & \\
\hline & CGRC.044 & Metodologia do Ensino da Educaçào de Jovens e Adultos & 54 & 4 & & & \\
\hline & CGRC. 47 & Traballho de Conclusäo de Curso & 81 & 5 & & & \\
\hline
\end{tabular}

Figura 1Matriz Curricular do Curso de Licenciatura em Geografia do IFPE. FONTE: http://portal.ifpe.edu.br/Beehome/resources/cont/storage/idPublic/ODkyOTsxNDE1OTkOMDAzMDAw 


\begin{tabular}{|c|c|c|}
\hline 411 & 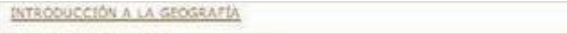 & \\
\hline 412 & MATEYATICA ESBECAAL & \\
\hline 413 & Geogaris risica I & \\
\hline 424 & GSOGRATH MUMAMA GEveEAS & \\
\hline 415 & SARTOCESAISA & \\
\hline \multicolumn{3}{|c|}{ UNA (1) MATERIA OPTATIVA A, a elegir entres } \\
\hline A11 & HESTCGIA $\triangle$ BGENTISA GENEAAL & \\
\hline A12 & HISTEQU BOCHAL CONTEMAOASSE & \\
\hline \multicolumn{3}{|l|}{;EGUNDOASTO } \\
\hline 41A & 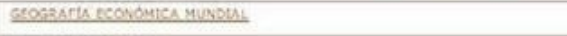 & $4 \mathrm{An}$ \\
\hline 416 & GHOGBATL RTSICA II & 413 \\
\hline 417 & GEOCRACH DE IA BOSLACION & 414 \\
\hline 418 & GEOCRATh De LOS ESPACIOS MARTITMOS & 423 \\
\hline 419 & MOGBCGerath & 413 \\
\hline \multicolumn{3}{|c|}{ UNA (1) MATERIA OPTATIVA B, a elegir entre: } \\
\hline A22 & BSCONOMta: & \\
\hline A23 & 5OCROCOCGA GESRAL & \\
\hline \multicolumn{3}{|l|}{ ERCER ASO } \\
\hline 421 & 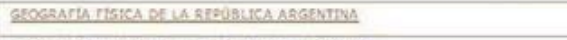 & $416-419$ \\
\hline 422 & 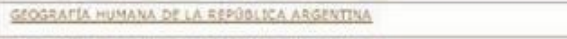 & 44 \\
\hline 431 & 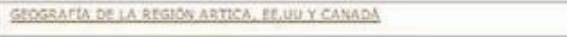 & $614-41 \mathrm{~A}$ \\
\hline 433 & GEOGSACHA DE EUROEA Y EUSE & $414-42 A$ \\
\hline 451 & CUNOAMENTOS DE UA EDUCACTON & \\
\hline 452 & 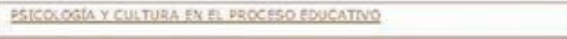 & 451 \\
\hline 424 & 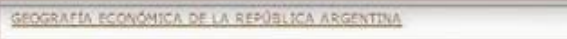 & $41 \mathrm{~A}$ \\
\hline 432 & GECGBATIA DE ASUA, ARBICA Y OCEANIS & $414-414$ \\
\hline 434 & GEOGRATU DE AMERRCA UTTMA & $422-124$ \\
\hline 453 & 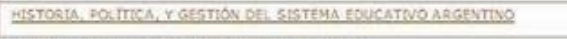 & 451 \\
\hline 454 & GEMTVAROO SODRE ESGESANZA DEVA GLOGQATHA & $452-453$ \\
\hline A32 & Ditroouccion A u rivesorts & \\
\hline A.33 & SOCBOESTADTENCA & \\
\hline A34 & TECNICAS DE ARALISIS ESPACCML & \\
\hline A.35 & TECANOCOTA EDUCATINA & \\
\hline $\mathbf{A 3 6}$ & COTATIVA C (Consignar nombre de la materia) & \\
\hline \multicolumn{3}{|l|}{ xubvto aso } \\
\hline 425 & 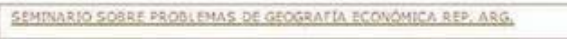 & $422-424$ \\
\hline 423 & 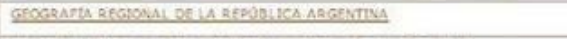 & $421-422-424$ \\
\hline 441 & 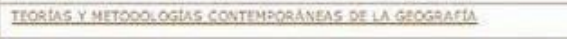 & $4: 1$ \\
\hline 455 & 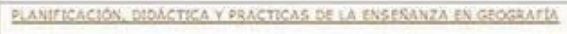 & 454 \\
\hline
\end{tabular}

Figura 2Matriz curricular do curso de Professorado em Geografia da FAHCE (UNLP). FONTE: http://www.fahce.unlp.edu.ar/academica/Areas/geografa/Carreras/profesoradoengeografa

\section{Conclusões}

Este estudo buscou verificar as estruturas que definem o curso de Licenciatura em Geografia em duas instituições de ensino superior, situadas em países diferentes, e os resultados apurados e exibidos acima atendem à demanda existente. Apesar das divergências, já citadas, podemos ver que algumas matérias são equivalentes nos dois cursos. Além desses fatores, é preciso levar em conta que enquanto no Brasil são realizados semestres, na Argentina são quadrimestres; e algumas 
matérias são anuais, e outras quadrimestrais na Argentina. Baseado no estudo realizado, foi montada uma tabela mostrando algumas disciplinas que possuem equivalência em ambos os cursos.

\begin{tabular}{|l|l|}
\hline \multicolumn{1}{|c|}{ IFPE } & \multicolumn{1}{|c|}{ FAHCE (UNLP) } \\
\hline FUNDAMENTOS DA CIÊNCIA GEOGRÁFICA & INTRODUCCIÓN A LA GEOGRAFIA \\
\hline HISTÓRIA DO PENSAMENTO GEOGRÁFICO & GEOGRAFIA HUMANA GENERAL \\
\hline CARTOGRAFIA BÁSICA & CARTOGRAFÍA \\
\hline GEOGRAFIA ECONÔMICA & GEOGRAFÍA ECONOMICA MUNDIAL \\
\hline GEOGRAFIA DA POPULAÇÃO & GEOGRAFÍA DE LA POBLACIÓN \\
\hline BIOGEOGRAFIA & BIOGEOGRAFÍA \\
\hline ANTROPOLOGIA CULTURAL & $\begin{array}{l}\text { ANTROPOLOGIA CULTURAL Y SOCIOLOGIA } \\
\text { (OPTATIVA) }\end{array}$ \\
\hline FUNDAMENTOS PSICOLÓGICOS DA EDUCAÇÃO & $\begin{array}{l}\text { PSICOLOGÍA Y CULTURA EN EL PROCESO } \\
\text { EDUCATIVO }\end{array}$ \\
\hline GEOGRAFIA E CULTURA & GEOGRAFÍA CULTURAL \\
\hline INTRODUÇÃO A FILOSOFIA & INTRODUCCIÓN A LA FILOSOFÍA (OPTATIVA) \\
\hline
\end{tabular}

\section{Referências}

BRASIL. Ministério da Educação. Escassez de professores no Ensino Médio: propostas estruturais e emergenciais. Brasília: Câmara de Educação Básica do Conselho Nacional de Educação, 2007.

FREIRE, Paulo. Pedagogia da autonomia: saberes necessários à prática educativa. São Paulo: Paz e Terra, 1996.

IFPE. Plano Pedagógico do Curso de Licenciatura em Geografia. Recife: CGEO, 2013.

SANTOS, Milton. Por uma outra globalização: do pensamento único à consciência universal. $11^{\mathrm{a}}$ ed. Rio de Janeiro: Record, 2004.

SAVIANI, Demerval. Escola e Democracia. Edição Comemorativa. Campinas: Autores Associados, 2008. 112p (Coleção Educação Contemporânea).

UNLP. Plan de Estudios del Curso de Professorado Em Geografia. La Plata: FAHCE, 2004. 